\title{
De la caricature à la peinture
}

Le portrait en rupture un exemple : la Bardot selon Saura

\section{Martine Heredia}

\section{(2) OpenEdition}

Journals

Édition électronique

URL : http://journals.openedition.org/agedor/2289

DOI : 10.4000 /agedor.2289

ISSN : 2104-3353

Éditeur

Laboratoire LISAA

Référence électronique

Martine Heredia, «De la caricature à la peinture », L'Âge d'or [En ligne], 4 | 2011, mis en ligne le 12 février 2019, consulté le 12 juin 2020. URL : http://journals.openedition.org/agedor/2289; DOI :

https://doi.org/10.4000/agedor.2289

Ce document a été généré automatiquement le 12 juin 2020.

L'Âge d'or. Images dans le monde ibérique et ibéricoaméricain 


\title{
De la caricature à la peinture
}

\author{
Le portrait en rupture un exemple : la Bardot selon Saura
}

\section{Martine Heredia}

Plusieurs historiens de l'art, parmi lesquels Gombrich et Werner Hofmann, ont mis en lumière les affinités entre la caricature et l'art moderne, montrant comment, en particulier, les expressionnistes de la fin du XIX siècle ont intégré, dans leurs distorsions, la caricature à l'art. Pour prolonger la réflexion au $\mathrm{XX}^{\mathrm{e}}$ siècle, je voudrais analyser les rapports qu'entretient la caricature, cette fois-ci, avec l'Expressionnisme Abstrait. Pour l'illustrer sous l'angle de l'activité créatrice, j'ai choisi d'interroger une partie de l'œuvre d'Antonio Saura, que certains critiques tiennent comme représentatif de ce courant artistique en Espagne. Ainsi, après avoir analysé le principe commun qui réunit caricature et peinture - c'est-à-dire la déformation -, il s'agira de montrer que si l'intention diffère, la peinture opère en plus un écart, en passant du difforme à l'informe.

\section{Un principe commun : la déformation}

2 La déformation, on le sait, est présente très tôt dans l'art : en atteste déjà l'art médiéval et, pour ce qui est du domaine hispanique, les distorsions du Greco ou les portraits de Goya. Mais elle atteint son apogée avec l'Expressionisme qui trouve, dans cette forme d'écriture artistique, le moyen de rendre compte d'un désordre symbolique. Si les expressionnistes de la fin du XIX ${ }^{e}$ siècle et du début du suivant ont tracé la voie en rejetant l'art bourgeois et ses artifices, en le niant par une explosive destruction des images et des formes, s'ils ont voulu révéler une autre réalité plus conforme aux angoisses existentielles des artistes du moment, c'est bien dans la continuité de cette rupture que s'engagent les expressionnistes abstraits de la seconde moitié du $\mathrm{XX}^{\mathrm{e}}$ siècle. Les premiers réagissaient violemment, en instaurant une vision nouvelle, comme une réponse à donner aux transformations du monde moderne et aux nouvelles découvertes scientifiques; les seconds cherchent une autre expression, un nouveau sens à donner à l'art, après les charniers et autres abominations révélées par la seconde guerre mondiale et, ainsi, renoncent à toute iconographie rassurante. Pour faire le lien 
entre les deux formes d'Expressionnisme, je rapprocherai deux œuvres significatives : au célèbre Cri de E. Munch (1893), me semble répondre parfaitement le tableau d'Antonio Saura, Gris $n^{\circ} 7$ (1959) ${ }^{2}$ qui, parfois, est intitulé Le Cri par la critique, confusion ou lapsus révélateur d'une parenté inévitable. Chez le peintre norvégien, sous fond de mer soulevée et sous un ciel rougeoyant, hurle un être hagard se serrant les tempes à deux mains, cri tragique de l'horreur existentielle. Dans ce visage, déformé jusqu'à devenir grimace, sont inscrites l'angoisse et la solitude de l'homme au sein d'une nature qui ne console pas mais qui répercute le cri au-delà du fjord et jusqu'au ciel couleur rouge sang. L'ensemble est grossi par l'emphase dramatique du style, une gamme chromatique violente qui, plus tard, prendra le nom de fauve et, surtout, un faisceau de lignes qui tend à produire une image déformée. Chez l'artiste espagnol, dans une palette réduite à l'extrême, qui décline les blancs, les noirs et les gris, une figure à mi-chemin entre l'humain et l'animal s'étire sur toute la surface de la toile, dans une tension qui désarticule le corps ${ }^{3}$. La bouche s'est transformée en museau, l'exacerbation déformante est produite par les membres écartelés. De fait, dans les deux cas, la démesure est à la hauteur d'un monde disloqué. Le rapprochement entre les deux œuvres est révélateur d'une attitude commune face au temps, face à la vanité odieuse de l'instant, face à la conscience de la finitude humaine. Le cri expressionniste est, selon Murielle Gagnebin, ce par quoi « l'homme agit, et simultanément, représente pour ainsi dire, sa révolte ${ }^{4}$ ", ce que les peintres mettent en scène en représentant l'homme comme un être conscient de sa propre hideur. Cependant, faut-il n'y voir que protestation ou rage provocatrice? On tend souvent à considérer l'Expressionnisme moins comme un style que comme un courant exprimant le sentiment d'une génération montante. Or, à plus de soixante ans d'écart entre les deux courants, subsiste la même violence dans la composition et le traitement des formes, la même exagération de hachures du trait, développant une esthétique de la brutalité et de la laideur.

C'est pour comprendre l'apparition du laid et de l'outrance dans l'art, que s'établit le lien avec la caricature. Le premier point commun est une même déviation par rapport à la norme académique et au culte de la beauté. Werner Hofmann définit la caricature comme le premier maillon d'une série de métamorphoses, d'une protestation contre le grand art et montre que l'avènement de la caricature, dont la spécialité est la déformation du modèle, a coïncidé avec l'éclosion de l'expressionnisme : le laid est considéré désormais comme expressif et s'impose comme l'autre facette de la réalité. La transgression que représente la caricature, admise dorénavant comme un art établi, libère de la tyrannie de l'idéal en s'éloignant des règles académiques, en rejetant l'imitation. Cette évolution conduisant peu à peu à une représentation non-figurative $\mathrm{du}$ monde, les expressionnistes abstraits de la deuxième moitié $\mathrm{du} \mathrm{XX}^{\mathrm{e}}$ siècle s'inscrivent parfaitement dans cette filiation. Il faut rappeler qu'ils sont passés, pour la plupart, par le surréalisme à partir duquel, on le sait, l'effroyable n'appartient plus à la caricature, mais glisse vers la peinture. Ce qui semblait impossible à représenter devient réalisable, et pour détruire la Beauté, on défigure le modèle.

Alors, comment ne pas être tenté d'illustrer métaphoriquement cette mise en pièces du

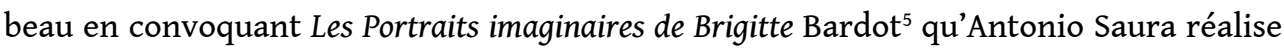
dès 1958 ? Dans la lignée de La Goulue et autres vedettes qui ont inspiré l'art de Toulouse Lautrec et qui peuvent se désarticuler comme un pantin, Saura s'attaque à un symbole de la culture populaire des années cinquante, faisant coexister la norme et sa déviation, l'idéal de beauté et son contre-idéal. En effet, alors que deux ans auparavant, 
en 1956, Vadim avait créé le mythe BB avec «Et Dieu créa la femme », faisant de Brigitte Bardot un monstre sacré, Saura le déconstruit et, en le désacralisant, crée un véritable monstre, autre face d'une même image que j'analyserai plus loin.

5 C'est que le deuxième point commun entre la caricature et la peinture expressionniste abstraite gravite, évidemment, autour du genre du portrait. Selon une même remise en cause du rapport entre réalité et mode de représentation, le modèle est malmené et sa copie défigurée. Alors que le rôle du portrait classique est de donner du modèle une vision flatteuse, dans le cas de la caricature et de la peinture le modèle est descendu de son piédestal et la distorsion dont il fait l'objet passe par une parenté formelle et technique. La liberté de la main étant une donnée essentielle, le caricaturiste dessine sous une impulsion, se laissant guider par son instinct. Cette liberté s'oppose, une nouvelle fois, à la main contrôlée et disciplinée du Beau idéal. Rien n'est moins vrai pour les artistes de l'après-guerre - et pour Saura en particulier - qui, dans le droit fil de cet héritage, revendiquent le geste rapide, irréfléchi, dans toute sa spontanéité, se réclamant pareillement d'un monde "à rebours » où la beauté et la raison n'imposent plus leur ordre. Il s'agit d'oublier ce qui a été appris, de retrouver en quelque sorte un degré zéro du graphisme. Ce monde à rebours est celui des origines, qui renvoie à l'enfance de l'art. D'où l'importance de la rapidité des gestes, leur répétition machinale ou rythmique, pour retrouver autant l'instantanéité de l'invention que la gratuité du geste ou le plaisir de «faire des traits ». Participent alors à la défiguration l'épaisseur du trait, l'emploi minimal de la couleur, la nature du support, en vue de lacérer la forme.

\section{Une autre finalité}

6 Cependant, le rapprochement entre la caricature et la peinture a ses limites. En d'autres termes, si le rejet du Beau et la déformation du modèle réunissent les deux formes d'art, l'intention diffère : en effet, la peinture de Saura, tout d'abord, se situe audelà de la reconnaissance et donc de l'imitation; en cela elle s'oppose totalement à la caricature; ensuite, si elle ne donne pas la ressemblance, elle ne communique pas davantage de message satirique, ni d'intention humoristique.

7 La différence est que, tout d'abord, la caricature étant la représentation chargée d'une personne d'après nature, d'une personnalité précise, elle est conçue essentiellement pour que le public reconnaisse l'objet, en visant d'abord l'effet de surprise. Pour cela, elle renforce les traits déjà visibles, fait ressortir les éléments marquants du physique ou du caractère en les isolant, grossis comme à la loupe, dans une déformation volontaire et consciente de la réalité. Il faut bien reconnaître que l'art du caricaturiste a quelque chose de diabolique au sens où « il révèle des déformations qui existent à l'état de velléité $»^{6}$. Si nous considérons quelques caricatures consacrées à l'actrice Brigitte Bardot, on remarquera que le plus souvent, à partir d'une expression graphique réduite au simple trait de crayon sur papier blanc, les éléments ciblés sont les cheveux, la bouche et les seins, critères de féminité. Ou alors, dans une version plus élaborée et une palette plus colorée ${ }^{7}$, l'accent est mis sur les mêmes détails, mais la déformation est obtenue à partir de l'exagération des courbes, la suggestion forcée des formes proéminentes (lèvres, seins, fesses) liées à la sexualité, l'écrasement des lignes du visage pour emphatiser le regard lascif, qui font que le spectateur reconnaît la vedette des années cinquante mais, surtout, le sex-symbol. Par le mouvement qui altère les 
proportions de la bouche, qui rétrécit le front et la ligne des yeux, le caricaturiste fait grimacer son modèle en fixant les expressions normalement mouvantes du visage, en plis stupidement contractés. C'est que, dirait Bergson, le comique naît aussi parce que le caricaturiste oppose à la grâce la raideur plus que la laideur. Enfin, dans d'autres versions $^{8}$, si la chevelure a perdu de son importance, c'est pour céder le terrain à la poitrine qui occupe les trois-quarts de l'espace et qui, avec les lèvres démesurément charnues, ajoute à la sensualité une mise en scène de la domination que renforce la ligne des sourcils froncés; autrement dit, ce n'est plus le modèle de la femme sexuellement libérée, mais soumise, de «En cas de malheur » (1958) de Claude AutantLarra, mais la dominatrice - et castratrice - de "Viva Maria " (Louis Malle, 1965) que le spectateur s'amuse à reconnaître. En amplifiant les indices, le caricaturiste déforme la beauté et tourne en dérision l'objet sexuel, dans l'intention de faire sourire. Car, le ressort de la caricature est bien, le plus souvent, le comique. Rappelons que Picasso luimême avait pratiqué la caricature de façon récréative et sa caricature d'autoportrait en forme de singe ${ }^{9}$ montre à quel point il reprenait le bestiaire cher aux caricaturistes à des fins humoristiques et d'autodérision.

Chez Saura, il n'est pas question de sourire et encore moins de rire; et s'il fallait parler d'humour, ce serait celui d'André Breton que Saura a fréquenté pendant ses années passées en France, quatre ans auparavant (1954-1955), cet humour noir et corrosif que les surréalistes manipulaient avec délectation. Si la caricature obéit bien à un processus dont le terme est la reconnaissance, la démarche qui anime Antonio Saura est inverse. Il s'empare du modèle comme d'une matière première et, alors que Vadim avait construit le mythe, Saura s'emploie à le déconstruire pour retourner à la figure de la Femme, thème récurrent dans sa trajectoire artistique de 1949 à $1997^{10}$. Pour l'artiste espagnol, il s'agit moins de s'intéresser au personnage lui-même, que de s'en servir comme d'un répertoire à sa disposition qui lui permet d'en faire quelque chose de différent, une image réinventée. Le fait est qu'avec BB, Saura s'attaque à une icône, celle du sexe-symbole des années cinquante, qui remplace la pin- up qu'avant lui, Willem de Kooning avait déconstruite en s'en prenant à un autre monstre sacré, Marilyn Monroe, qu'il rend tragiquement monstrueuse. C'est que la figure de la Femme a quelque chose de l'idole, symbole à la fois maternel et érotique dans laquelle, comme le dit lui-même Saura, se réunissent la Vierge, la Mère et la Putain. Nous sommes loin de la sensualité des peintures académiques, loin des schémas attendus repris par la caricature, loin de toute ressemblance, loin de toute intention satirique; Saura ne cherche pas non plus à remettre en question le culte que le public a voué à la vedette ${ }^{11}$. Nous sommes plus près de Bataille; Saura trouve dans le modèle ce que le philosophe français avait mis en relation avec le plaisir et la souffrance, avec l'érotisme et la mort. Malmener, salir, renvoient au sexe ;

9 «Plus grande est la beauté, plus profonde est la souillure " $^{12}$, écrit Bataille, ce qui, chez le peintre espagnol, se traduit dans le rapport violent et corporel entre le geste et la matière. Saura cherche dans l'acte de peindre de quoi libérer sa créativité ; être, dit-il, face à la toile et concevoir « le tableau comme un lit où l'on fait l'amour ou comme un champ de bataille $»^{13}$. 


\section{Du difforme à l'informe} qu'entre ses mains, l'icône devient matrice, se transformant en structure primaire, dans un combat qui réunit geste et matière. Ici, au-delà d'une volonté expressive, l'artiste dépasse le difforme pour rejoindre l'informe; c'est pourquoi il me semble que le terme d'expressionniste abstrait est moins approprié pour Antonio Saura que celui de peintre de l'informel, comme je l'ai développé dans ma thèse. De l'écriture automatique des surréalistes, il a gardé le geste irréfléchi, dont il vise la gratuité pour atteindre, dans ce voyage à rebours, l'instantanéité de l'invention qui caractérise l'enfance. Ce rapport à la peinture est nettement visible dans le portrait de Brigitte Bardot datant de $1962^{14}$ parce qu'il rappelle que le bonhomme est l'image matrice du graphisme enfantin. Aussi, la figure qui en résulte est-elle présentée frontalement, mise à plat, aplatie sur la surface où fond et forme se rejoignent, mettant sur le devant de la scène une production qui est de l'ordre du manuel plus que du visuel mais qui, surtout, donne à voir un élan échappant au contrôle de la raison. La prédominance de la tête, la proéminence des yeux, le barbouillage des lignes renvoient au gribouillage de l'enfant, activité sans objet défini, liée au plaisir et participant de la prise de possession de l'espace.

11 Cependant, loin de caricaturer le dessin d'enfant, bien au-delà de ce qui serait pure régression, c'est par la manipulation des matériaux et en atteignant cet état d'expérience élémentaire que l'artiste espagnol compte repartir pour créer plus librement, en dehors de tout « conditionnement» culturel. Pour cela, tout au long de son œuvre, il pervertit la forme, défigurant le modèle - en l'occurrence ici Brigitte Bardot, mais ailleurs il s'agira de Philippe II, de Goya ou de Rembrandt -. De cette façon, il se l'approprie en forçant les formes, en conduisant les traits du corps et du visage jusqu'au paroxysme, dans un processus continu de destruction-construction, de violence du geste et du rythme, dont l'aboutissement est le dévoilement de la matière en voie de métamorphose. Le corps dont on devine les seins, soulignés dans la partie inférieure par un trait maladroit qui ne referme même pas le cercle - comme peut le faire le petit enfant, à un certain stade de son développement ${ }^{15}$ - et le visage de BB ne sont qu'amas de peinture, de taches, de coulures. Mais c'est surtout sur la tête que se fait voir le travail et que se lit la violence du geste dans une dynamique avec la matière picturale. Sur une toile de grand format $(250 \times 200 \mathrm{~cm})$, par un processus de gestes qui s'enchaînent, où les décisions, selon Saura lui-même ${ }^{16}$, sont instantanées et les corrections à peine possibles, les signes sont détournés : la bouche et les yeux, symboles de la sensualité de $\mathrm{BB}$ croquée par les caricaturistes, défigurent le visage en laissant voir dents et orbites qui transforment le sex-symbol en monstre, à mi-chemin entre la femme et la bête. Ainsi, Saura brouille les limites entre le beau et le laid qui oscillent entre l'attraction et la répulsion, entre amour et destruction, car le peintre, écrit Saura, «face à l'impossible beauté, est semblable à l'amant qui à force d'aimer finit par transformer en monstre l'objet de son amour $»^{17}$. Pas de chair, pas de viande non plus au sens où Deleuze l'entend quand il parle de Bacon, mais un amas de pâte que Saura manipule, en l'étirant ici avec son pinceau, en l'écrasant là avec ses doigts, en éclaboussant là encore la toile, de sorte que l'ensemble ne soit surtout pas parfait. Guidé par la vie de la matière fuyante, Saura assiste, en quelque sorte, à la forme spontanée de son œuvre. L'objet de désir se transforme en pur objet esthétique, faisant 
glisser la sensualité du monde de la chair du modèle à celui de la toile où le peintre se confronte à la matière, donnant corps à ses fantasmes ou à ses visions. Et si, enfin, la chevelure, autre référence à la sensualité soulignée par les caricaturistes, n'est pas représentée dans sa liberté sauvage, mais ramassée en un petit chignon qui ne manque pas de rappeler les danseuses de Degas, c'est pour mieux montrer, sans doute, que ce qui importe n'est pas la fidélité au modèle - et donc à la ressemblance - mais la liberté du peintre face à l'art. Le résultat obtenu est, comme l'écrit Saura à propos des autoportraits, « un monstre pictural $\aleph^{18}$, qui dérange par l'ironie de sa mise en œuvre et en déconcerte plus d'un, parce que Saura met non seulement à mal la traditionnelle fonction du portrait, mais aussi celle de la caricature. De fait, le portrait implique une double relation, celle du rapport au modèle et celle au sujet à qui il est présenté, autrement dit le spectateur. Il sous-entend alors le problème de l'identification. C'est donc là que s'arrête la parenté entre caricature et peinture. Antonio Saura nous montre que la ressemblance n'a rien à voir avec la reconnaissance. En remettant en question la ressemblance, en évacuant la fonction de rappel du portrait et de la caricature, Saura rend manifeste que le monstre ne renvoie pas au modèle mais au tableau lui-même. En mettant l'observateur face au non reconnaissable, le portrait inquiète parce qu'il interroge sur l'identité. Quand il détruit la " figure ", Saura détruit l'illusion du portrait et, avec elle, le fondement même de la représentation, l'image se situant au-delà des apparences. Alors que le portrait est censé être transparent, Saura en souligne au contraire l'opacité, mettant ainsi l'accent sur la fragilité de l'image elle-même et sur la dialectique qu'introduit le jeu des miroirs entre le réel et l'imaginaire. Car, selon le titre qu'il donne à la série des tableaux de Brigitte Bardot, il s'agit bien de "portraits imaginaires » et non de simples portraits. Saura nous donne à voir, une "chair ", au sens où l'entend Merleau-Ponty, c'est-à-dire cette dimension matricielle de l'image, qui effectue une métamorphose qui est celle du travail de l'imaginaire et de l'imagination.

Donc, pour conclure sur les liens qui réunissent caricature et peinture dans leur traitement de la déformation du portrait, ces exemples de portraits de Brigitte Bardot montrent que lorsque la déformation s'intègre dans une vision personnelle, elle dépasse l'intention propre de la caricature, lorsque la destruction est au cœur du projet artistique, elle devient le moteur de la création et un exercice pour la recherche plastique, et ainsi, le résultat n'appartient plus à la caricature. Saura opère une distorsion de l'image pour laisser surgir celle qu'il ne connaît pas encore, parce qu'il travaille à réinventer la peinture.

\section{NOTES}

1. SAURA, Antonio, Portrait imaginaire de Brigitte Bardot, 1959. Óleo sobre lienzo. 250 x $200 \mathrm{cms}$. Museo Abstracto de Arte Español. Cuenca.

2. SAURA, Antonio, Gris $n^{\circ} 7,1959$, huile sur toile, 250 x $200 \mathrm{~cm}$, Centro de Arte Reina Sofía, Madrid.

3. http://www.artehistoria.jcyl.es/arte/obras/12317.htm (Les liens renvoient à des sites de particuliers qui ont intégré les œuvres de Saura auxquelles je fais référence). 
4. GAGNEBIN, Murielle Fascination de la laideur, [1978), Seyssel, édit. Champ Vallon, 1994, p.172.

5. SAURA, Antonio, Portrait imaginaire de Brigitte Bardot, 1959. Óleo sobre lienzo. 250 x $200 \mathrm{cms}$. Museo Abstracto de Arte Español. Cuenca.

6. BERGSON, Henri, Le rire, [1940], Paris, PUF, 1995, p. 20.

7. http://brigittebardot.canalblog.com/tag/art

8. http://www.wittygraphy.com/picture/show/1013310/brigitte-bardot

9. PICASSO, Pablo, Autoportrait en forme de singe, Paris, 1903.

10. HEREDIA, Martine, L'art informel en Espagne: d'une praxis à la formulation d'une expérience du monde, Thèse de doctorat sous la direction de Nancy Berthier, Paris, Université Paris-Est, LISAA, EA 4120, 2009.

11. SAURA, Antonio, Note book (memoria del tiempo), Valencia, Artes Gráficas Soler, 1992, p. 69 : « Ha querido verse una intención satírica contra el culto de la vedette y la alienación del público frente a ella, desconociéndose el todopoderoso deseo plástico y [...] la verdad que se oculta bajo ciertas formas de venganza y la complejidad de la fascinación del mito, quedando desprovista de todo fundamento la sospecha de una persona repulsa. »

12. BATAILLE, Georges, L'érotisme, [1957], Paris, Minuit, 1979, p.161.

13. SAURA, Antonio, Del gesto a la acción, in Tropos, n³-4, Madrid, 1972, p.12.

14. http://www.flickr.com/photos/sebastian-silva/2145616058/

15. HEREDIA, Martine, L'art informel en Espagne: d'une praxis à la formulation d'une expérience du monde, op.cit.

16. SAURA, Antonio, Note book (memoria del tiempo), op.cit., p.55.

17. Ibid. p. $45:$ : [...] el pintor, frente a la imposible belleza, se asemeja al amante que de tanto amar acaba convirtiendo en monstruo el objeto de su amor ».

18. SAURA, Antonio, Ibid. p. 28.

\section{RÉSUMÉS}

C'est pour comprendre l'apparition du laid et de l'outrance dans la peinture que s'établit le lien avec la caricature. Ces deux formes d'expression se rejoignent dans le traitement fait au portrait, la transgression libérant de la tyrannie de l'idéal. Comment ne pas être tenté alors d'illustrer métaphoriquement cette mise en pièces du Beau en convoquant Les Portraits imaginaires de Brigitte Bardot ${ }^{1}$ qu'Antonio Saura réalise dès 1958 ? Après les avoir confrontés avec des caricatures de l'actrice, il s'agit ici de montrer que le rapprochement entre la caricature et la peinture a ses limites. La peinture de Saura se situe au-delà de la reconnaissance et donc de l'imitation; le peintre espagnol s'empare du modèle comme d'une matière première et l'icône devient matrice, se transformant en structure primaire, dans un combat qui réunit geste et matière. Au-delà d'une volonté expressive, l'artiste dépasse le difforme pour rejoindre l'informe.

Al intentar entender la aparición de lo feo y lo excesivo en la pintura, es cuando se establece el vínculo con la caricatura. Estas dos formas de expresión se reúnen en el tratamiento del retrato, luego que la transgresión libera de la tiranía. Así que, ¿cómo resistir a la tentación de ilustrar metafóricamente ese descuartizar de lo Bello ante los Retratos imaginarios de Brigitte Bardot que Antonio Saura realizó en 1958? Después de cotejar los retratos y unas caricaturas de la actriz, se trata aquí de mostrar que el paralelo entre la caricatura y la pintura tiene sus límites. La pintura de Saura se sitúa más allá de la identificación y pues de la imitación; el pintor aragonés se 
apodera del modelo como si fuera una materia prima, con lo cual el icono se convierte en matriz, transformándose en una estructura primaria, mediante un combate que reúne gesto y materia. Más allá de una mera voluntad expresiva, el artista deja atrás lo disforme para alcanzar lo informe.

INDEX

Mots-clés : Saura, peinture espagnole du XXe siècle, caricature, informel

Palabras claves : Saura, pintura española $\mathrm{XX}^{\circ}$, caricatura, informal

\section{AUTEUR}

MARTINE HEREDIA

Crimic EA 2561, Université Paris-Sorbonne 